\title{
ILCEA
}

Revue de l'Institut des langues et cultures

d'Europe, Amérique, Afrique, Asie et Australie

37 | 2019

Des genres en Méditerranée : pratiques,

représentations et transfert

\section{Quand les lettrés chinois rencontrent la civilisation moderne : le monde méditerranéen dans les écrits chinois à la fin de la dynastie des Qing (fin XIX ${ }^{\mathrm{e}}$ début $\mathrm{xx}^{\mathrm{e}}$ siècle)}

When Chinese Scholars Meet Modern Civilisation: The Mediterranean World in Chinese Writings at the End of the Qing Dynasty (late $19^{\text {th }}$ - early $20^{\text {th }}$ century)

Félix Jun Ma

\section{OpenEdition}

Journals

Édition électronique

URL : http://journals.openedition.org/ilcea/7372

DOI : 10.4000/ilcea.7372

ISSN : 2101-0609

Éditeur

UGA Éditions/Université Grenoble Alpes

Édition imprimée

ISBN : 978-2-37747-099-0

ISSN : $1639-6073$

\section{Référence électronique}

Félix Jun Ma, «Quand les lettrés chinois rencontrent la civilisation moderne : le monde méditerranéen dans les écrits chinois à la fin de la dynastie des Qing (fin $x \mid x^{\mathrm{e}}$ - début $\mathrm{xx} \mathrm{e}^{\mathrm{e}}$ siècle) », ILCEA [En ligne], 37 | 2019, mis en ligne le 04 novembre 2019, consulté le 15 janvier 2020. URL : http://

journals.openedition.org/ilcea/7372 ; DOI : 10.4000/ilcea.7372

Ce document a été généré automatiquement le 15 janvier 2020.

(C) ILCEA 


\section{Quand les lettrés chinois rencontrent la civilisation} moderne : le monde méditerranéen dans les écrits chinois à la fin de la dynastie des Qing (fin XIX ${ }^{\mathrm{e}}$ - début $\mathrm{XX}^{\mathrm{e}}$ siècle)

When Chinese Scholars Meet Modern Civilisation: The Mediterranean World in Chinese Writings at the End of the Qing Dynasty (late $19^{\text {th }}$ - early $20^{\text {th }}$ century)

Félix Jun Ma

1 À la fin des Qing (1644-1912), dernière dynastie chinoise, entre les années 1840 et 1860, la Chine a fait l'amère expérience d'une rencontre brutale avec le monde occidental au travers des guerres de l'opium : la première contre les Anglais entre 1840 et 1842 et la seconde contre l'alliance anglo-française entre 1856 et 1860 . Les deux défaites humiliantes subies par l'empire chinois face aux Puissances occidentales ont ébranlé la vision sinocentrique de l'empire céleste, vision selon laquelle la civilisation chinoise était supérieure à toute autre. La Chine est alors forcée de reconnaitre qu'il n'est désormais plus possible de considérer les Occidentaux, bien plus avancés en matière de technologies, comme des barbares non-civilisés. Mais l'échec des réformes économiques puis institutionnelles entamées dans le but d'égaliser la puissance de l'Occident à la fin des Qing plonge ce pays, riche d'une histoire millénaire et d'une tradition bien ancrée dans la vie politique et sociale, dans une crise sans précédent face à la modernité occidentale.

Dans ce contexte, après les guerres de l'opium, entre les années 1860 et 1900, les premiers voyageurs chinois de l'époque moderne se rendent en Europe occidentale, qu'ils atteignent en passant par la Méditerranée. La plupart de ces voyageurs sont des 
diplomates ou des missionnaires envoyés par la cour impériale chinoise pour établir des relations officielles avec les pays du Vieux Continent, d'autres sont plutôt des intellectuels qui se rendent en Europe pour diverses raisons dans le cadre de voyages personnels. Mais, quel que ce soit leur statut, ce sont tous des lettrés formés aux enseignements confucéens et capables de réfléchir à la situation de la Chine face à la menace de l'Occident. Ils sont donc obligés de se positionner et de développer de nouvelles conceptions autour des différentes problématiques de leur temps, telle la construction de l'État-nation et de la modernité chinoise, par le biais de la comparaison avec l'Occident, dont la Méditerranée est une porte d'entrée territoriale.

3 À l'époque, en effet, la Méditerranée est le passage obligé pour les voyageurs chinois se rendant en Europe par voie maritime. Son nom apparaît donc dans tous les récits de voyage chinois vers le Vieux Continent. Si le terme Xihai 西海 (Mer de l'Ouest) a été employé pendant des siècles par les lettrés chinois pour faire spécifiquement référence à la mer située entre l'Empire romain et le Royaume séleucide, celui de Dizhonghai 地中 海 a commencé à le remplacer sous les Ming (1368-1644), afin de traduire de façon plus juste le sens de "Méditerranée $»^{1}$. Ce mot signifie littéralement «la mer entre les terres", et fait plus précisément référence à cette mer située entre l'Europe et l'Afrique ${ }^{2}$. Au-delà des simples descriptions de mer ou de paysages, les comparaisons que les voyageurs chinois font entre la civilisation chinoise et les cultures de cette zone géographique s'avèrent ainsi particulièrement significatives de leurs préoccupations $\mathrm{du}$ moment, entre histoire et modernité. Que nous apprennent donc les écrits chinois de la fin des Qing sur les relations entre ces deux mondes bien distincts? Comment la Méditerranée telle qu'ils l'ont observée et décrite a-t-elle aidé des auteurs chinois à bâtir leurs réflexions sur la modernisation politique de la Chine?

4 Soulignons d'abord que les récits de voyage de la fin des Qing ont déjà fait l'objet de nombreuses études approfondies, en particulier dans le monde académique chinois. Plusieurs ouvrages et recueils publiés à ce sujet méritent d'être mentionnés. L'historien Zhong Shuhe choisit et commente plus de cent récits ou journaux de voyage à l'étranger publiés entre 1840 et 1912. Sa première collection, qui se compose de dix volumes, est parue dans les années 1980 (Zhong éd., 1985), tandis que la seconde, de cinquante-cinq volumes, voit le jour en 2016 (Zhong éd., 2016) ${ }^{3}$. Ces grandes collections sont souvent utilisées par les chercheurs comme corpus principal. À partir de celles-ci, par exemple, Yin Dexiang s'intéresse à la découverte de la vie culturelle de l'Occident par les émissaires chinois et à leurs réflexions sur la construction de l'identité culturelle chinoise (Yin, 2009), tandis que Li Ya met l'accent sur les idées véhiculées à travers les récits de voyage de la fin des Qing aux premières années de la République qui ont pu influencer la création de l'État-nation chinois (Li, 2011).

5 Récemment, les sinologues occidentaux et japonais ont également montré un intérêt particulier pour les itinéraires des premiers diplomates chinois à l'étranger et pour leurs journaux de voyage, mais leurs sujets de recherche sont restés relativement vastes. Si Feng Chen-Schrader privilégie une étude comparative entre les civilisations chinoise et occidentale (Chen-Schrader, 2004), Jenny Huangfu Day « intègre l'histoire diplomatique et intellectuelle dans les analyses littéraires et les sciences de la communication» (Day, 2018). Dans le même temps, trois auteurs japonais prêtent attention à la prise de conscience des diplomates chinois quant au nouvel ordre mondial, au-delà du seul universalisme chinois, et à l'impact de ces derniers sur la diplomatie moderne, à travers une étude minutieuse sur les différentes versions de ces 
récits de voyage et sur la diffusion et la réception de ces derniers parmi les lettrés chinois (Okamoto et al., 2014).

6 Par ailleurs, certains classiques de la sinologie qui ont fait date sur la réception de la modernité chez de grands réformateurs chinois à la fin des Qing méritent aussi d'être mentionnés ici. Ils évoquent également les voyages de ces derniers en Occident et au Japon. Benjamin Schwartz, dans son In Search of Wealth and Power: Yen Fu and the West, retrace le séjour de Yan Fu 严复 (1854-1921) en Grande Bretagne entre 1877 et 1879 et analyse comment sa pensée a été influencée par les idées politiques occidentales (Schwartz, 1964). Dans Liang Ch'i-ch'ao and Modern Chinese Liberalism, Philip Huang souligne l'importance du rôle joué par les grands philosophes japonais sur la pensée 《libérale» de Liang Qichao 梁启超 (1873-1929), exilé au Japon pendant quatorze ans entre 1898 et 1912 (Huang, 1972). Hsiao Kung-chuan mentionne aussi, dans A Modern China and a New World: K'ang Yu-wei, Reformer and Utopian, 1858-1927, les voyages de Kang Youwei 康有为 (1858-1927) au Japon, en Europe et en Amérique, voyages qui ont contribué à la réflexion du penseur sur la modernité (Hsiao, 1975).

7 Mais, parmi tous les travaux mentionnés ci-dessus, aucun ne considère le monde méditerranéen comme un objet spécifique de recherche. Il est tout à fait légitime, pourtant, de dire que c'est à travers la Méditerranée que les Chinois ont commencé à découvrir pour la première fois la civilisation moderne et sont entrés en contact direct avec la modernité dans les années $1860^{4}$. Pour les lettrés chinois comme pour l'historien contemporain, établir ce lien entre la Chine et la Méditerranée permet non pas de mettre en évidence les différences entre les mentalités chinoises et occidentales, mais de mieux comprendre les tensions qui se dégagent, d'ouest en est, entre tradition et modernisation. À cette fin, concentrons-nous sur trois pays du monde méditerranéen qui ont rarement fait l'objet de recherches parmi les historiens de la civilisation chinoise, alors qu'ils sont considérés comme des berceaux des civilisations méditerranéennes, anciennes et modernes : l'Égypte, l'Italie et la Grèce.

\section{L'Égypte : un premier regard sur la modernité}

Pour arriver en Europe par voie maritime, les voyageurs chinois devaient passer par l'Égypte, porte d'entrée vers la Méditerranée. Tous ont exprimé leur admiration pour la civilisation égyptienne. En 1866, Binchun 斌椿 (1804- ?), le premier mandarin chinois envoyé en Occident par la cour impériale des Qing, traverse le Nil pour visiter les pyramides à côté du Caire. Il décrit les paysages spectaculaires de ce pays dans son court récit Chengcha biji 乘槎笔记 [Note de voyage], le premier récit de voyage d'un mandarin chinois en Égypte (Binchun, 1985 : 105). À peine dix ans plus tard, en traversant le pays pour se rendre en Europe, les collègues de Guo Songtao 郭嵩奉 (1818-1891), premier ambassadeur chinois en Occident $^{5}$, achètent des tableaux reproduisant des sites historiques d'Égypte et commencent à s'intéresser à l'écriture hiéroglyphique égyptienne. Guo enregistre cette anecdote dans son Shixi jicheng 使西纪 程 [Mission diplomatique en Occident] (Guo, 1985 : 74-75). En Europe, il discute aussi de la culture et de la civilisation de l'Égypte antique avec des égyptologues.

9 Par ailleurs, dans les écrits de voyage des premiers diplomates chinois, l'Égypte est non seulement ancienne, mais aussi moderne. Certes, l'histoire de "Masr» (traduit phonétiquement par les Chinois de l'époque comme Maixi 麦西) les passionne, avec ses pyramides, son iconographie et ses coutumes séculaires, mais c'est aussi dans ce pays 
qu'ils ont leur première vision de la modernité, notamment à travers le Canal de Suez et le chemin de fer.

Le Canal de Suez est une réalisation française dont les travaux se terminent en 1869. Il permet de relier la Méditerranée et la mer Rouge. Lorsque les premiers Chinois à destination de l'Europe arrivent en Égypte entre les années 1860 et 1880, tous sont saisis d'admiration devant cette construction miraculeuse. Zhang Deyi 张德蓕 (1847-1918) par exemple, qui accompagne Binchun pour visiter l'Égypte en 1866 et revisite ce pays quatre ans après avec le commissaire impérial Chonghou 崇厚 (1826-1893) en tant qu'interprète d'anglais ${ }^{6}$, qualifie ce grand ouvrage d'« extraordinaire ». C'est pour lui la preuve que "l'homme peut vaincre la nature » (Zhang, 1985 : 363), l'incarnation de la légende chinoise de Yu Gong. Dans celle-ci, Yu Gong, bien que très âgé, est parvenu à déplacer les montagnes entravant la route qui séparait les champs de sa maison ${ }^{7}$. Le journal de voyage du deuxième ambassadeur chinois en France, Zeng Jize 曾纪泽 (1839-1890), donne un autre exemple de cette même réaction : « Le Français De Lesseps a réussi à creuser un canal à la frontière entre l'Europe et l'Afrique pour que les bateaux circulent librement. Cet homme a réduit de dix mille kilomètres le trajet entre l'Asie et l'Europe, c'est un exploit brillant !» (Zeng, 1985 : 145). Impressionnés par cette construction réalisée grâce aux sciences et technologies modernes, ces lettrés chinois se montrent singulièrement sensibles à la modernité occidentale. Liu Xihong 刘锡鸿 (1822 ou 1823-1891?), premier ambassadeur adjoint chinois en Grande-Bretagne et premier ambassadeur chinois en Allemagne, attribue ainsi ce succès à la modernisation commerciale de l'Occident :

Chaque fois que les Occidentaux réalisent de grands travaux, ils créent leur société par actions [...]. Lorsqu'ils creusent un souterrain dans les montagnes pour ouvrir une rivière, font des recherches sur l'univers, fabriquent des objets extraordinaires, ouvrent de nouveaux ports, ils ont recours à cette méthode [...] (Liu, $1985: 62$ ).

11 Toujours en Égypte, certains voyageurs, dont Liu Xihong, ont vu le train pour la première fois. Si les chemins de fer se sont developpés rapidement en Europe dans la première moitié du XIX ${ }^{e}$ siècle, la Chine n'a eu son premier train qu'en 1876, à Shanghai. La même année, en Égypte, Liu Xihong voit et prend le train pour la première fois de sa vie. Dans son journal, écrit-il, « même les trains de petite vitesse peuvent rouler à plus de cent kilomètres par heure, quelques jours suffisent pour arriver à une destination qui se trouve à cinq mille kilomètres d'ici. C'est une technologie ingénieuse [...]» (ibid., 62-63). Pour autant, selon Liu, cette modernité n'est pas souhaitable en Chine: il considère que la construction ferroviaire reste inadaptée aux circonstances nationales ${ }^{8}$. En premier lieu parce qu'elle engagerait trop de dépenses tandis que la tradition confucéenne préconise une vie frugale. Le sens de la frugalité, d'après Confucius, est une qualité essentielle pour gouverner un pays. En second lieu parce que les techniques et les technologies occidentales ne sont employées que pour fabriquer des objets, les $q i$ (器), seulement fonctionnels et pratiques, selon les enseignements confucéens, alors que la priorité d'un pays devrait être de retrouver la bonne voie de gouvernement (dao 道), pour atteindre la parfaite bonté. A contrario, si un pays se concentre sur l'apprentissage pratique, et non sur les principes de bonne gouvernance, c'est qu'il a "renversé le bon ordre de l'univers " ${ }^{9}$. Pour Liu Xihong, la civilisation matérielle de l'Occident ne peut pas perdurer : elle devrait s'inspirer du confucianisme pour corriger la voie qu'elle a suivie jusqu'à présent.

12 Face à la modernité, les voyageurs chinois de la fin des Qing, tels que Liu Xihong, ont en somme des sentiments assez complexes. Tout en étant fascinés par les travaux réalisés 
grâce aux sciences et technologies modernes, ils sont souvent hésitants quant à l'introduction de ces dernières en Chine, car la tradition chinoise considère le développement matériel et technologique comme un objectif secondaire. Pour ces lettrés, la civilisation chinoise restant supérieure aux autres civilisations du monde, le seul écart entre la Chine et l'Occident n'est que d'ordre pratique et il suffira, le moment venu, de connaître les techniques utilisées par les Occidentaux pour que l'Empire céleste retrouve sa prospérité.

Cependant, la profonde crise politique et économique qui agite la Chine des Qing durant la seconde moitié du XIX siècle l'oblige à faire des concessions à la modernité et à abandonner progressivement des éléments traditionnels jugés inadaptés aux temps modernes. Dans cette démarche, certains lettrés chinois sont aussi amenés à repenser non seulement la tradition mais aussi et surtout la compatibilité entre la civilisation occidentale et la culture chinoise. Kang Youwei, grand réformateur politique mais farouche opposant à la révolution de la fin des Qing, en est un exemple extraordinaire ${ }^{10}$ ; son Voyage en Italie [Yidali youji 意大利游记] est particulièrement intéressant sur ce point.

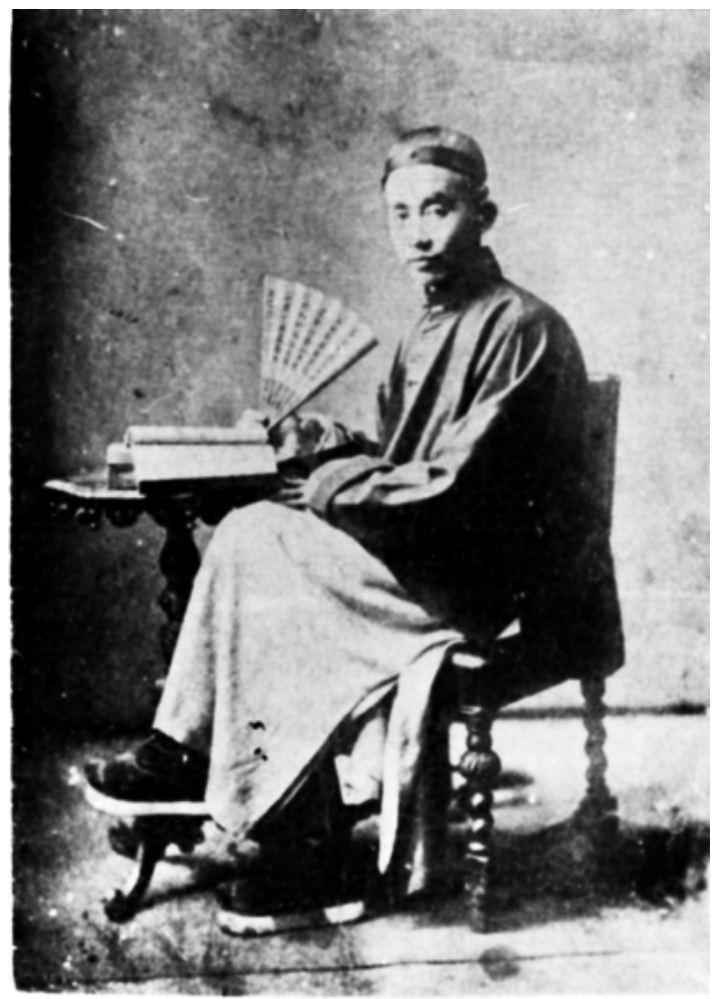

Zhang Deyi 张德彝 $(1847-1918)^{11}$ 


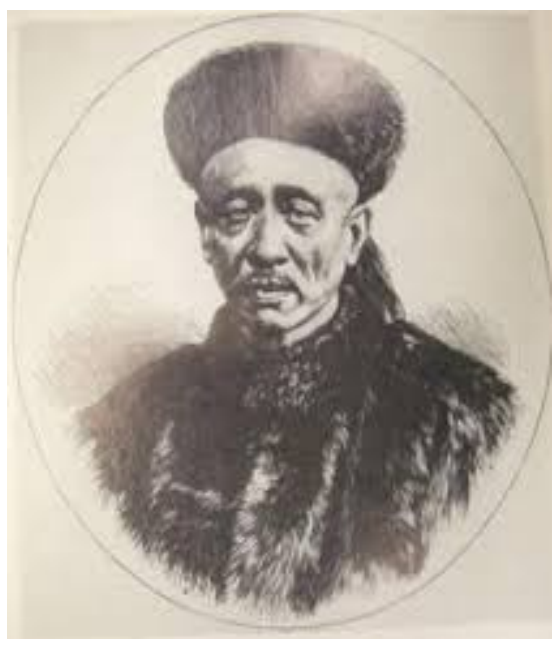

Liu Xihong 刘锡鸿 (1822 ou 1823 - ? $)^{12}$

\section{L'Italie : éloge et critique de la modernité}

14 Il est notable qu'à première vue, l'Italie ne laisse pas une bonne image aux voyageurs chinois de la fin des Qing. Parfois, elle peut même être considérée comme un symbole de pauvreté et de sous-développement ${ }^{13}$. Par exemple, lorsqu'il visite Naples en 1886, Zou Daijun 邹代钧 (1854-1908), géographe connu de l'époque, trouve que les mendiants et les voleurs y sont trop nombreux dans les rues. Il note même que peu d'Italiens ont reçu une bonne éducation (cf. Casalin, 2012: 33). De son côté, le grand penseur Wang Tao 王韬 (1828-1897), alors qu'il voyage en Méditerranée et fait escale à Naples, en 1879 , observe des Italiens monter à bord de son bateau pour exécuter des chants afin de gagner leur vie (Wang, $1985: 657)^{14}$. Zhang Deyi constate de son côté que les Italiens ne se douchent même pas une fois par mois (Zhang, $1985: 370$ ) ! En 1904, l'année où il se rend en Italie, Kang Youwei fait les mêmes remarques (Kang, 1985 : 70-71 et 74), estimant même que l'Italie est le pays le plus pauvre des grands pays européens et que sa situation économique n'est pas plus enviable que celle de l'Empire chinois (ibid., 73 et 179).

Comment expliquer cette impression globale de grande pauvreté ? Zhang Deyi et $\mathrm{Li}$ Shuchang黎庶昌 $(1837-1896)^{15}$ considèrent que la paresse du peuple en est la principale cause (ibid., et Li, 1985 : 518). Mais Xue Fucheng 薛福成 (1838-1894) ${ }^{16}$ conclut que l'Italie a dû dépenser trop d'argent dans le développement de ses forces militaires, ce qui a conduit à une augmentation considérable des dettes de l'État (Xue, 1985 : 307).

Lors de son voyage de 1904, Kang Youwei ne cesse toutefois de chanter les louanges de la Méditerranée, berceau des brillantes civilisations de l'Antiquité, telles que l'Égypte antique, les Cités grecques, ou la Rome antique. Durant son voyage en Méditerranée à destination de l'Italie, en 1904, il immortalise ainsi par écrit les sentiments profonds qui le saisissent:

[Le bateau] vogue sur la Méditerranée vers 6h. [...] Cela me fait penser à Alexandre le Grand, aux exploits brillants de Hannibal, à la civilisation phénicienne, cela me fait aussi imaginer des navires de guerre d'il y a plusieurs milliers d'années, [...] J'ai conçu un poème oralement: "La mer entre les terres est tout comme le Lac du paradis chinois, elle a nourri les fils chéris du Ciel et créé des miracles [...] ». (Ibid., 66) 
L'auteur a également composé un «Chant de la Méditerranée 》 (Dizhonghai ge 地中海 歌) dans lequel il présente non seulement la situation géographique et les différentes civilisations historiques de la région, mais émet aussi l'idée d'un déterminisme environnemental, susceptible d'expliquer la particularité politique et culturelle du monde méditérranéen. D’après Kang Youwei, en effet, la géographie spécifique de cet espace territorial et maritime n'y favorise guère la centralisation du pouvoir. D'où par exemple la naissance des anciennes Cités-États italiennes, fondées sur les échanges commerciaux, dont la tradition politique préconisait la liberté au lieu du despotisme. Kang s'enthousiasme :

Je ne cesse de m'exclamer durant mon voyage. Les Chinois, qui ont toujours connu une civilisation agricole et une grande unité politique et culturelle, ne sauraient imaginer les différences entre les diverses formes d'État et de gouvernement des pays méditerranéens et celle de la Chine. Toutes les pensées innovantes, les différents régimes politiques et les diverses traditions des Européens ont vu le jour grâce à la Méditerranée. (Ibid., 165)

À partir de cette hypothèse, Kang montre que « les parlements en Europe ont été créés grâce à la situation géographique de la Méditérranée » (ibid., 144), qui rend possible la décentralisation et le partage du pouvoir. À l'inverse, la Chine n'a jamais inventé son propre régime parlementaire, non pas $d u$ fait «que le peuple chinois n'est pas intelligent, mais parce que la géographie de son pays ne le permet pas» (ibid., 140). En somme, remarque-t-il, étant donné leurs différentes situations géographiques, l'Empire chinois et l'Occident ont eu deux évolutions totalement opposées : la Chine a connu une longue période paisible d'unité nationale mais s'est dégradée du fait de l'absence de compétitivité au niveau international, tandis que le monde méditerranéen a connu un âge sombre, rempli de guerres et de conflits, mais a finalement engendré une civilisation moderne (ibid., 161). En conclusion, la modernité ne serait donc qu'occidentale, la Chine ne pouvant que l'assimiler partiellement, au risque d'y perdre sa propre tradition.

En ce qui concerne la modernisation politique, Kang Youwei insiste par conséquent sur la greffe du système parlementaire européen en Chine, système qu'il conçoit comme à l'origine de la puissance occidentale et comme l'avenir de tous les États de la planète. Mais en même temps, il faut rester très vigilant, remarque-t-il, car ce système représentatif est un produit unique du monde méditerranéen. Puisque ce dernier favorise l'établissement de petites entités politiques indépendantes dans la région et ne privilégie pas le pouvoir absolu du souverain, les forces sociales pourraient ainsi se développer contre les autorités locales et centrales. Mais la Chine doit tirer leçon de l'expérience de l'Europe pour se prémunir de tout déchirement de la nation. L'Italie, qui se trouve au centre de la Méditerranée, bénéficie naturellement de la diversité politique et culturelle de cette région, mais elle n'est devenue un État unifié qu'à partir de la seconde moitié du xixe siècle. Dans ce sens, Kang Youwei loue la grandeur de Camillo Cavour (1810-1861), acteur principal de l'unité italienne, pour ses talents en diplomatie et sa bravoure qui ont permis d'éviter une révolution sanglante dans son pays. Selon l'auteur, pour prévenir une destruction totale de la nation et conserver son unité nationale, la Chine doit ainsi se garder de toute tendance à la décentralisation et à la sécession. Considérant que les conditions nationales de l'Italie sont similaires à celles de la Chine, Kang propose donc que son propre pays suive la voie de cet État pour se moderniser économiquement et politiquement (ibid., 174). 
Par ailleurs, Kang Youwei rappelle aux Chinois qu'il n'est pas nécessaire de flatter l'Occident en toute chose et qu'il faut rester très fiers d'être les héritiers de Confucius. Dans son récit de voyage, par exemple, s'il dédie de nombreux passages à la description des monuments religieux qu'il a visités en Italie, il n'hésite pas à émettre des commentaires négatifs sur le christianisme. En fait, il se méfie de cette religion qui risque, selon lui, de favoriser l'expansion des impérialistes occidentaux qui se disputent alors les intérêts militaires et économiques dans le monde entier. Pour Kang, le christianisme ne peut être directement appliqué à la Chine; en effet, que cette religion exclusive déclenche des conflits militaires au nom du «salut des êtres humains » est pour lui incompréhensible (ibid., 184) ${ }^{17}$. À l'inverse, le confucianisme est selon lui parfaitement capable de remplacer le christianisme puisque la pensée confucéenne est plus tolérante et laisse faire les individus. Elle peut ainsi remédier aux maux de la civilisation occidentale, comme l'indique Kang Youwei, qui s'efforce alors d'ériger le confucianisme en religion d'État en Chine.

Pour conclure, il apparaît d'un côté que Kang Youwei est en admiration devant les civilisations méditerranéennes et la modernité occidentale. Critique vis-à-vis des défauts de la civilisation chinoise, il est convaincu que la Chine, comme l'Italie, devra se moderniser aux niveaux politique et économique. Mais, d'un autre côté, il s'oppose vivement à une occidentalisation totale de la Chine: pour construire une identité nationale chinoise, il faut d'abord et avant tout vénérer le confucianisme ${ }^{18}$. En comparant la Chine avec l'occident et la tradition chinoise avec la modernité occidentale, il manifeste autant un intérêt spontané que le souci de la construction d'un État-nation chinois moderne, capable de rivaliser avec les Puissances occidentales dans la compétition internationale. C'est dans cette même perspective et dans le souci d'alimenter son nationalisme culturel, que Kang Youwei commente la civilisation grecque, dans son Voyage en Grèce [Xila youji 希腊游记], écrit en 1908.

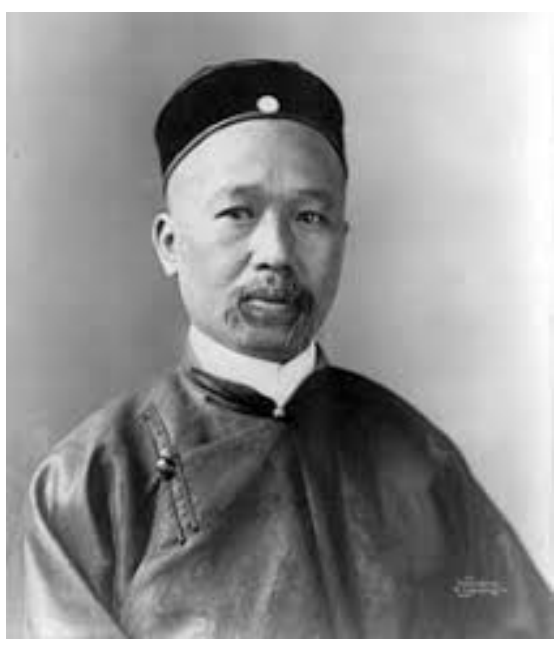

Kang Youwei 康有为 (1858-1927) ${ }^{19}$

\section{La Grèce : construction de la modernité politique}

La Grèce n'est pas un pays très fréquenté par les voyageurs chinois à la fin des Qing. Malgré son histoire extrêmement riche, elle n'est pas considérée comme l'un des plus grands pays européens et n'a donc pas suscité une attention particulière chez les intellectuels chinois de l'époque. Son nom même n'est pas souvent mentionné dans les 
récits de voyage chinois. Le texte de Kang Youwei reste peut-être le seul récit de la fin des Qing spécifiquement consacré au monde hellénique. À travers ce voyage en Grèce, Kang Youwei s'approprie les éléments historiques et culturels du pays pour repenser la modernisation politique de l'Empire céleste et fonder un nationalisme chinois moderne.

Dans son récit, dans un premier temps, Kang Youwei fait l'éloge de la grandeur d'Athènes : cette cité grecque est à l'origine de toute la civilisation européenne, qui a elle-même influencé des milliers de nations du monde entier (Kang, 2007 : 458). Les concepts modernes tels que les "droits du peuple», la "république», ou la « démocratie » viennent tous de la Grèce antique : selon Kang, comme il en avait déjà eu l'intuition pour la Rome antique en 1904, cela tient notamment à la situation géographique de cette dernière en Méditerranée, situation favorable à la naissance des différentes cités et des différentes cultures politiques. Bien que la Chine possède sa propre civilisation depuis des milliers d'années, remarque-t-il, pour autoriser sa modernisation politique, elle devra ainsi adopter les institutions européennes, comme le système parlementaire et le constitutionnalisme, qui sont en réalité des inventions d'Athènes.

Cependant, il tend à nuancer quelque peu cet élan d'affiliation politique au modèle athénien. Pour lui, il n'y a pas de solution absolue en tout lieu et en tout temps. Il conjecture ainsi que l'acceptation des notions politiques modernes issues du monde grec et la construction du système représentatif ne sont possibles pour tous les pays du monde dans le présent de son époque qu'à la seule condition qu'ils développent leur propre modernité sur le modèle de l'Occident (ibid., 461). Il insiste donc sur le fait qu'au lieu d'envisager un régime politique idéal pour tous les États, il faut plutôt penser les circonstances particulières de chaque pays suivant sa propre singularité. Ce penseur se focalise ainsi sur la question de l'identité.

Sur le plan culturel, Kang observe notamment que la population locale attache toujours une importance particulière à la conservation du patrimoine architectural et des objets anciens pour préserver l'histoire et la culture de son pays, alors que la Chine n'a nullement cette conscience et a tendance à détruire ses antiquités, ce qui correspond pour lui à un archétype de la barbarie ${ }^{20}$. Il avance ainsi cette suggestion, au sujet de la protection du patrimoine culturel :

La Chine est connue comme un pays civilisé possédant un vaste territoire, mais, aujourd'hui, tous les palais impériaux construits depuis plus de mille ans ont disparu, tandis que cette petite cité [Athènes] a bien conservé un certain nombre de monuments qui ont survécu plus de deux mille ans, n'est-ce pas un élément précieux dans la culture grecque ? (Ibid., 463)

En somme, Kang insiste sur l'importance de l'histoire et de la culture dans l'identité d'une nation: le patrimoine culturel est un héritage à transmettre car il éveille la mémoire collective de ce que Benedict Anderson qualifie de "communauté imaginée " (Anderson, 2006). Si la Chine n'est point consciente de la responsabilité qu'elle a de le sauvegarder, elle risque de perdre des supports matériels puissants pour aider à la construction de la nation moderne chinoise.

Par ailleurs, la comparaison entre les trajectoires historiques de la Grèce et de la Chine est employée par Kang Youwei pour créer un sentiment de fierté nationale et contribuer au rétablissement de la supériorité de la civilisation chinoise, qui doit retrouver son essor dans les temps modernes. De fait, lorsque Kang Youwei visite la 
Grèce en 1908, il est en réalité déçu par ce qu'il observe concrètement : des bâtiments délabrés, des nuages de poussières, des arbres desséchés et des herbes sèches (Kang, 2007 : 459). Il explique ainsi que ce pays est en déclin évident par rapport à la Grèce antique qu'il a pu imaginer. De plus, note-t-il, les Grecs modernes n'ont rien de comparable aux anciens Grecs, qui ont déjà totalement disparu, étant donné que leur civilisation a subi une destruction totale à cause des invasions étrangères. En revanche, souligne Kang, la civilisation chinoise, ancienne et brillante, elle aussi, reste la seule civilisation du monde à avoir pu conserver tout au long de l'histoire une continuité sinon au niveau de son patrimoine matériel, au moins au niveau culturel et institutionnel (ibid., 466). En d'autres termes, la Chine doit suivre le modèle occidental, mais elle a évidemment son propre potentiel pour se moderniser à partir de sa propre histoire et de sa propre tradition, forgeant ainsi l'identité nationale chinoise. Son retard dans la modernisation par rapport à l'Occident n'est que temporaire.

Pour mettre en perspective ces réflexions comparatives de Kang Youwei, on peut aussi mentionner le travail intellectuel de Liang Qichao, un de ses disciples et lui aussi un réformateur célèbre de la fin des Qing, qui s'intéresse également à l'histoire de la Grèce antique pour enrichir sa pensée en faveur d'un nationalisme chinois moderne. Dans ses différents écrits, il s'inspire notamment de l'histoire de Sparte pour appeler les Chinois à cultiver un esprit martial et une obéissance aux lois sans condition, éléments essentiels non seulement pour garantir l'ordre social, mais aussi pour renforcer un sentiment d'appartenance au sein du peuple. Comme il le dit, dans le projet de la modernisation politique, «Sparte est le premier remède à la Chine ! (Liang, 1936:2). Encore une fois, tout en repensant la modernité occidentale, les lettrés chinois se préoccupent du destin politique de leur pays.

\section{Conclusion}

Dans notre article, nous avons essayé d'établir des liens entre les civilisations méditerranéennes et la modernisation politique de la Chine de la fin des Qing. En prenant l'Égypte, l'Italie et la Grèce comme des exemples particuliers et en travaillant sur des écrits chinois à propos de ces pays, nous avons pu observer comment des aspects historiques anciens et modernes de ces pays méditerranéens ont permis à des lettrés chinois de l'époque de repenser la tradition et de réfléchir sur la construction de la modernité en Chine. Ces lettrés, qui se soucient de la place de la Chine sur la scène internationale face à l'Occident et de la sortie de la crise politique et sociale de leur pays, n'hésitent pas à comparer la civilisation chinoise aux civilisations méditerranéennes pour définir une identité chinoise et trouver un remède à une Chine qui a du mal à se moderniser. Mais leur attitude envers la modernité occidentale peut être autant admirative que critique. Admirative, parce que l'Occident montre sa domination dans la technologie et les institutions ; critique, parce que la Chine a besoin de se valoriser pour maintenir sa confiance en elle, et ne pas devenir une civilisation inférieure au monde occidental. Depuis la seconde moitié du XIX ${ }^{\mathrm{e}}$ siècle, les civilisations méditerranéennes sont ainsi devenues progressivement pour la Chine une référence incontournable en matière de modernisation. C'est à partir d'une telle référence que les intellectuels chinois ont le plus souvent découvert la modernité et tenté de modifier leur façon de voir le monde. 


\section{BIBLIOGRAPHIE}

ANDERSON Benedict (2006), Imagined Communities: Reflections on the Origin and Spread of Nationalism (dernière édition), Londres, New York : Verso Corporation.

CASALIN Federica (2012), « Jianlun wan Qing youji zhong de Yidali xingxiang - yi bijiao wenxue xingxiangxue de lilun wei zhongxin 简论晚清游记中的意大利形象 - 以比较文学形象学的理论 为中心 » [De l'image de l'Italie dans le récits de voyage de la fin des Qing - du point de vue de l'imagologie en littérature comparée], Wakumon或問, 22, 25-38.

CHEN Shiru 陈室如 (2011), «Wan Qing yuwai youji de haiyang shuxie - yi Zhang Deyi “Gaoben Hanghai shuqi huibian” weili 晚清域外游记的海洋书写一以张德犀《稿本航海述奇汇编》为例 》 [Les océans dans les récits de voyage à l'étranger de la fin des Qing - Étude de « Manuscrit de Mes incroyables expériences à l'étranger » de Zhang Deyi], Chengda zhongwen xuebao 成大中文学报 [Journal académique en chinois de l'Université de Cheng Kung], 33, 131-164.

CHEN-SCHRADER Feng (2004), Les diplomates chinois découvrent l'Europe (1866-1894), Paris : Hachette. CoHEN Paul (1987), Between Tradition and Modernity: Wang T'ao and Reform in Late Ch'ing China, Cambridge (U.S.) : Harvard University Press.

DAY Jenny Huangfu (2018), Qing Travelers to the Far West - Diplomacy and the Information Order in Late Imperial China, Cambridge : Cambridge University Press.

HSIAo Kung-Chuan (1975), A Modern China and a New World: K'ang Yu-Wei, Reformer and Utopian, 1858-1927, Seattle et Londres : University of Washington Press.

HUANG Philip (1972), Liang Ch'i-ch'ao and Modern Chinese Liberalism, Seattle et Londres : University of Washington Press.

KANG Youwei 康有为 (2007, première parution en 1908), « Xila youji 希腊游记 » [Voyage en Grèce], Kang Youwei quanji, dibaji 康有为全集·第八集 [CEuvres complètes de Kang Youwei, vol. 8] (compilé par Jiang Yihua et Zhang Ronghua), Pékin : Zhongguo renmin daxue chubanshe.

LI Ya 李涯 (2011), Diguo yuanxing - Zhongguo jindai lüwai youji yu minzu guojia jiangou 帝国远行 : 中 国近代旅外游记与民族国家建构 [Le long voyage de l'Empire - les récits de voyage à l'étranger dans la Chine moderne et la construction de l'État-nation], Pékin : Zhongguo shehui kexue chubanshe.

LIANG Qichao 梁后超 (1936, première parution en 1902), «Sibada xiaoshi 斯巴达小史》 [Annales de Sparte], Yinbingshi heji, zhuanji 饮冰室合集·专集 [Collection des œuvres du Studio du Buveur de glace, recueil d'ouvrages sur des sujets spécialisés, vol. 15] (compilé par Lin Zhijun), Pékin : Zhonghua shuju.

LIU Xihong 刘锡鸿 (1988), Liu Guanglu yigao 刘光禄遗稿 [Manuscrit posthume de Liu Xihong], Shen Yunlong (dir.), Taipei : Wenhai chubanshe.

MA Félix Jun (2017), « Guo Songtao : un diplomate chinois au carrefour de la tradition et de la modernité », Europeana, 10, 71-85.

MizoGuchi Yuzo 溝口雄三 (1999), Zuowei “fangfa” de Zhongguo 做为“方法”的中国 [La Chine comme « méthode »] (traduit par Lin Youchong), Taipei : Guoli bianyi guan. 
ОКАмото Takashi岡本隆司 et al. (2014), Deshi nikki no jidai - kyiyosue no chugoku to gaiko出使日記の 時代: 清末の中国と外交 [L'époque des carnets de diplomates - La diplomatie et la Chine de la fin des Qing], Nagoya : Nagoya daigaku shuppan'kai.

SCHWARTZ Benjamin (1964), In Search of Wealth and Power: Yen Fu and the West, Cambridge (U.S.) : Harvard University Press.

WEI Yuan 魏源 (1875, première parution en 1843), Haiguo tuzhi 海国图志 [Traité illustré des royaumes maritimes], Pingqing jinggu dao, Chine.

WoNG Young-tsu 汪荣祖 (2000), Zouxiang shijie de cuozhe : Guo Songtao yu Dao, Xian, Tong, Guang shidai 走向世界的挫折 : 郭嵩奉与道咸同光时代 [Vers le vaste monde avec frustration : Guo Songtao et la fin des Qing], Changsha : Yuelu shushe.

Xu Jiyu 徐继番 (2001), Yinghuan zhi lüe 瀛寰志略 [Abrégé des routes maritimes] (première parution en 1849), Shanghai : Shanghai shudian chubanshe.

YIN Dexiang 尹德叛 (2009), Donghai Xihai zhijian 东海西海之间 [Entre la Mer de l'Est et la Mer de l'Ouest], Pékin : Beijing daxue chubanshe.

ZHANG Yuquan 张宇权 (2004), Sixiang yu shidai de luocha - Wan Qing waijiaoguan Liu Xihong yanjiu 思 想与时代的落差 : 晚清外交官刘锡鸿研究 [L'écart entre pensée et époque : le cas de Liu Xihong, diplomate de la fin des Qing], Tianjin : Tianjin guji chubanshe.

ZHONG Shuhe 钟叔河 [dir.] (1985), Zouxiang shijie congshu 走向世界丛书 [Collection « voyages dans le monde "], vol. 1-10, Changsha : Yuelu shushe :

- Binchun 斌椿, Chengcha biji 乘嵄笔记 [Notes de voyage] (rédigé en 1866), vol. 1.

- Guo Songtao 郭嵩奉, Shixi jicheng 使西纪程 [Mission diplomatique en Occident] (rédigé en 1877), vol. 4.

- KANG Youwei 康有为, Yidali youji 意大利游记 [Voyage en Italie] (rédigé en 1904), vol. 10.

- Li Shuchang 黎庶昌, Xiyang zazhi 西洋杂志 [Miscellanées d'Occident] (rédigé entre 1876 et 1881), vol. 6.

- LIu Xihong 刘锡鸿, Yingyao siji 英轺私记 [Carnet de vie en Grande Bretagne] (rédigé entre 1877 et 1878), vol. 7.

- WANG Tao 王韬, Manyou suilu 漫游随录 [Récit de voyages d'agrément] (rédigé entre 1867 et 1870), vol. 6.

- XUE Fucheng 薛福成, Chushi Ying Fa Yi Bi siguo riji 出使英法义比四国日记 [Mission diplomatique en Grande Bretagne, en France, en Italie et en Belgique] (rédigé entre 1890 et 1891), vol. 8.

- ZHANG Deyi 张德彔, Suishi Faguo ji 随使法国记 [Dans l'entourage d'un diplomate en France] (rédigé en 1871), vol. 2.

- ZENG Jize 曾纪泽, Chushi Ying Fa E guo riji 出使英法俄国日记 [Missions diplomatiques en Grande Bretagne, en France et en Russie] (rédigé entre 1878 et 1886), vol. 5.

ZHONG Shuhe 钟叔河 (2010), Zouxiang shijie, Zhongguoren kaocha xifang de lishi 走向世界一中国人考 察西方的历史 [Parcourir le monde - expérience de l'Occident par les voyageur chinois] ( $3^{\mathrm{e}}$ éd.), Pékin : Zhonghua shuju.

ZHONG Shuhe 钟叔河 [dir.] (2016), Zouxiang shijie congshu xubian 走向世界丛书续编 [Collection «Voyages dans le monde» (suite)], vol. 1-55, Changsha : Yuelu shushe.

ZHU Yitian 朱忆天 (2016), “ “Wenming yizhi” lun yu Kang Youwei houqi sixiang de zhuanxiang “文明移植”论与康有为后期思想的转向》 [La théorie de la 《transplantation de civilisation » et l'évolution de la pensée de Kang Youwei durant la dernière moitié de sa vie], Wenshizhe 文史哲 [Journal of Chinese Humanities], 6, 72-83. 


\section{NOTES}

1. Ce nom figure déjà sur la Carte complète du monde entier [Kunyu wanguo quantu 坤舆万国全图] publiée en 1602, alors que sous les Qing, Xu Jiyu 徐继鈿 (1795-1873) indique dans son Yinghuan zhi lüe 灜寰志略 [Abrégé des routes maritimes] que Mei di de la nüe reste la transcription phonétique chinoise de "Méditerranée ».

2. Wei Yuan 魏源 (1794-1856) précise la situation géographique de la Méditerranée dans son Haiguo tuzhi 海国图志 [Traité illustré des royaumes maritimes] (Wei, 1875 : vol. 74, 1).

3. On peut retrouver ces commentaires sur les récits de voyage dans Zouxiang shijie: Zhongguo ren kaocha xifang de lishi 走向世界: 中国人考察西方的历史 [Parcourir le monde - expérience de l'Occident par les voyageurs chinois].

4. Nous ne mentionnerons pas ici le Japon, qui est un autre terrain où certains lettrés chinois ont fait cette même expérience, mais plus tardivement. Le Pays du Soleil Levant, qui se modernise profondément grâce à la réforme de Meiji à partir de 1868, ne deviendra un exemple de modernisation pour l'empire chinois qu'après la première guerre sino-japonaise (1894-1895) à l'issue de laquelle la Chine a connu une défaite écrasante.

5. Les études sur Guo Songtao sont assez nombreuses. Citons ici (Wong, 2000) et (Ma, 2017).

6. Au nom de l'empereur chinois, Chonghou avait alors pour mission d'aller présenter ses excuses devant Napoléon III, à cause du massacre d'un certain nombre de missionnaires français en Chine.

7. Pour une étude approfondie sur les écrits de Zhang Deyi sur les mers et les océans, voir (Chen, $2011: 131-164)$.

8. Liu Xihong adresse en 1881 un rapport à l'Empereur et à l'Impératrice au sujet des aspects négatifs de la construction de chemins de fer en Chine (Liu, 1988 : 71-108). Voir aussi (Liu, 1985 : 63).

9. Pour une analyse approfondie de la pensée de Liu Xihong, voir (Mizoguchi, 1999: 215-238) et (Zhang, 2004).

10. Pour une étude sur Kang Youwei et sa pensée, voir (Hsiao, 1975).

11. Source photo: < https://guoxue.ifeng.com/a/20180612/58681467_0.shtml>, consulté le 29 avril 2019.

12. Source image : <http://www.sohu.com/a/286830553_260616>, consulté le 29 avril 2019.

13. Sur la vision de l'Italie dans les récits de voyage de la fin des Qing, voir (Casalin, 2012 : 25-38).

14. Pour une analyse de la pensée de Wang Tao, voir (Cohen, 1987).

15. Li a accompagné Guo Songtao en Europe en 1877 et y a travaillé jusqu'en 1881 en tant qu'émissaire diplomatique.

16. Xue a été successivement ambassadeur en Grande Bretagne, en France, en Italie et en Belgique entre 1889 et 1894.

17. Ces critiques envers le christianisme sont largement partagées par les contemporains de Kang Youwei. Par exemple, Xue Fucheng, après avoir visité Rome en 1891, déclare que les Occidentaux sont trop ignorants pour avoir une croyance chrétienne (Xue, 1985 : 314).

18. Zhu Yitian définit la théorie de Kang Youwei comme une "transplantation de civilisation» (wenming yizhi 文明移植) qui lui paraît cependant paradoxale : en fin de compte, la supériorité de la civilisation chinoise l'emporte sur la civilisation occidentale universaliste. Notre propos va aussi dans ce sens. Voir (Zhu, 2016).

19. Source photo : <https://en.wikipedia.org/wiki/Kang_Youwei>, consulté le 29 avril 2019.

20. Kang mentionne aussi ce point dans son Voyage en Italie. 


\section{RÉSUMÉS}

À la fin de la dynastie des Qing, entre les années 1860 et 1900, les voyageurs chinois ont traversé la Méditerranée à destination de l'Europe pour des missions diplomatiques ou des voyages personnels. Ces lettrés confucéens, soucieux de l'avenir de l'Empire du Milieu en pleine crise, ont découvert la modernité dans le monde méditerranéen et ont commencé par conséquent à réfléchir sur la modernisation chinoise en se référant aux éléments historiques, culturels ou modernes des pays de la région. Leurs récits de voyage en Égypte, en Italie ou encore en Grèce montrent en fait la complexité de leur pensée face aux tensions entre modernité occidentale et tradition chinoise. C'est à partir de ces réflexions qu'ils ont cherché à moderniser la politique chinoise et à construire une identité moderne de la nation.

In the late Qing period, between the 1860s and 1900s, Chinese travelers crossed the Mediterranean to Europe for diplomatic missions or personal trips. These Confucian scholars, who were concerned about the future of the Middle Kingdom in crisis, discovered modernity in the Mediterranean world and therefore reflected on the Chinese modernisation by referring to the historical, cultural or modern elements of the countries of this region. In fact, their travel notes in Egypt, Italy and Greece, show the complexity of their thinking about the tensions between Western modernity and Chinese tradition. From these reflections, they sought to modernise Chinese politics and build a modern identity of the nation.

\section{INDEX}

Mots-clés : monde méditerranéen, modernité, modernisation politique, récits de voyage chinois, lettrés chinois confucéens, Égypte, Italie, Grèce, Chine de la fin des Qing (fin du xixe siècle - début du xxe siècle)

Keywords : Mediterranean world, modernity, political modernisation, Chinese travel notes, Chinese Confucian scholars, Egypt, Italy, Greece, late Qing China (late nineteenth century - early twentieth century)

\section{AUTEUR}

\section{FÉLIX JUN MA}

Univ Paul Valéry Montpellier 3, LLACS EA 4582, F34000, Montpellier, France 